\title{
Nested Array With Time-Delayers for Target Range and Angle Estimation
}

\author{
Wen-Qin Wang, Cong Ling \\ Department of Electrical and Electronic Engineering, Imperial College London, London SW7 2AZ, United Kingdom. \\ Email: w.wang@imperial.ac.uk; c.ling@imperial.ac.uk
}

\begin{abstract}
Since nested array with phased-array cannot directly estimate the range of targets due to range ambiguity, this paper proposes a nested array with diverse time-delayers for target range and angle estimation. The essence is to construct a new array structure by systematically nesting two uniform linear arrays through diverse time-delays which yields range-dependent receiving array beampattern. Using second-order statistics of the received data, it is capable of providing a dramatic increase of the system degrees-of-freedom which means more sources can be resolved. More importantly, target range and angle can be estimated by exploiting the range-dependent array beampattern. The performance improvements are evaluated by examining the Cramér-Rao lower bounds.
\end{abstract}

\section{INTRODUCTION}

The number of sources that can be resolved by a $N$ element uniform linear phased array is $N-1$. To overcome this constraint, multiple techniques [1], [2] have been proposed for detecting more sources than physical sensors. Minimum redundancy array is suggested in [1], but there are no closed form expressions for the array geometry and achievable degrees-offreedom (DOFs) for a given $N$ elements. In [3], the fourthorder cumulants are applied to increase DOFs, but its applicability is restricted to non-Gaussian sources. Multiple-input multiple-output (MIMO) can significantly increase DOFs, but it is not applicable to passive arrays.

Nested array [4], [5], which nests two or more uniform linear arrays (ULAs), is a promising technique to significantly increase the DOFs. It provides $O\left(N^{2}\right)$ DOFs using $N$ physical elements by exploiting the second-order statistics of the received data through difference co-array processing. Nested array has closed form expression for the array geometry and achievable DOFs for given $N$ elements, but only phased arrays are employed in the literatures. This limits its performance in mitigating undesired range-dependent interferences and thus limits its ability to resolve targets from noise and interference by adaptive beamforming. Frequency diverse array (FDA) offers range-dependent transmit beampattern by employing a small frequency increment compared to the carrier frequency across the array elements [6]. This range-dependent beampattern provides a potential to estimate target range, but only active transmitting FDA is presented in existing literatures.

Inspired by active FDA using diverse frequency increments to achieve range-dependent transmit beampattern, this paper proposes a new nested array with diverse time-delayers to achieve range-dependent receiving array beampattern for target range and angle estimation. The essence is to systematically nest two ULAs through diverse time-delayers. The use of time-delayers generates a receiving array beampattern that is a function of range, time, and angle. Moreover, the DOFs are increased by exploiting the second-order statistics of the received data through the difference co-array processing. In doing so, the targets can be localized in range and angle domains without sacrificing the advantage of nested array in increasing array DOFs.

This paper is organized as follows. Section II proposes the new nested array with diverse time-delayers. The signal model and corresponding difference co-array processing algorithm are presented. Section III is devoted to evaluate the performance of the designed nested array in target range and angle estimations. Finally, simulation results are provided in Section IV and concluding remarks are given in Section V.

\section{New Nested Array Signal and Data Models}

Like the basic nested array using phased arrays, the new nested array also is basically a concatenation of two ULAs through diverse time-delayers whereas they are not employed in the basic nested array, namely, the inner and outer arrays consist of $N_{1}$ and $N_{2}$ elements with interelement spacings $d_{1}$ and $d_{2}=\left(N_{1}+1\right) d_{1}$, respectively. More precisely it is a linear array with element locations given by the union of $S_{\text {iner }}=\left\{n_{1} d_{1}, n_{1}=1,2, \ldots, N_{1}\right\}$ and $S_{\text {outer }}=\left\{n_{2}\left(N_{1}+1\right) d_{1}, \quad n_{2}=1,2, \ldots, N_{2}\right\}$. Figure 1 illustrates the proposed nested array based on diverse time-delayers. Since it provides not only phase-dependent beampattern but also range-dependent beampattern, there are rather different characteristics in beampattern, signal model and direction-ofarrival (DOA) estimation from basic nested array.

We derive the proposed nested array receiver pattern in a rather general way by assuming the signal coming to the array is a linearly frequency modulated (LFM) waveform

$$
s(t)=\exp \left\{j 2 \pi\left(f_{0} t+\frac{1}{2} k_{r} t^{2}\right)\right\}
$$

where $f_{0}$ is the starting frequency (equal to the carrier frequency) and $k_{r}$ is the chirp rate. The signal related with the direction $\theta$ and range $r$ in far-field and received by the $n$th sensor can be modeled as

$$
x_{n}(r, \theta, t)=\frac{\sqrt{\sigma}}{r_{n}} s\left(t-\tau_{n}\right) \exp \left\{-j \frac{2 \pi f_{0}+k_{r}\left(t-\tau_{n}\right)}{c_{0}} r_{n}\right\}+v_{n}(t)
$$

where $r_{n}$ and $\tau_{n}$ denote the target range to the $n$th sensor and corresponding propagation time, respectively, $\sigma$ is the target reflection coefficient, $c_{0}$ is the speed of light, and $v_{n}(t)$ is the 


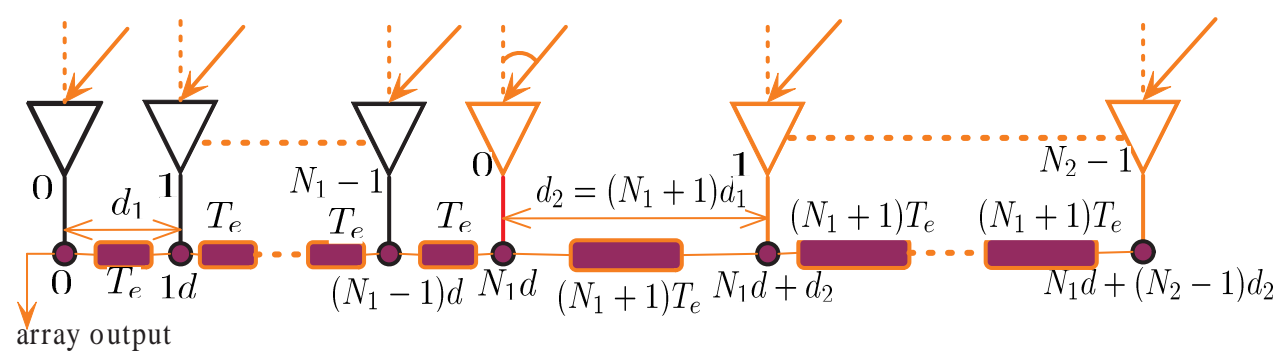

Fig. 1: Illustration of proposed nested array receiver based on diverse time-delayers.

noise. Here, $\tau_{n}=n T_{e}$ with $T_{e}$ being a fixed inter-element time delay and $r_{n}$ can be approximated as $r_{n}=r-n d \sin \theta$ with $d$ being the inter-element spacing.

In amplitude sense, $r_{n}$ can be replaced by $r_{0}$. We then have

$$
\begin{aligned}
x_{n}(r, \theta, t)= & \frac{\sqrt{\sigma}}{r_{0}} \exp \left\{j\left(2 \pi f_{0} t+\frac{k_{r} t^{2}}{2}-\frac{2 \pi f_{0} r_{0}}{c_{0}}-\frac{k_{r} r_{0} t}{c_{0}}\right)\right\} \\
& \times \exp \left\{j n \left(-2 \pi f_{0} T_{e}+\frac{k_{r} x_{n} T_{e}^{2}}{2}-k_{r} t T_{e}+\frac{2 \pi f_{0} d \sin \theta}{c_{0}}\right.\right. \\
& \left.\left.+\frac{k_{r} r_{0} T_{e}}{c_{0}}+\frac{k_{r} d \sin \theta t}{c_{0}}-\frac{k_{r} x_{n} d \sin \theta T_{e}}{c_{0}}\right)\right\}+v_{n}(t)
\end{aligned}
$$

Since $r_{0} \gg \frac{x_{n} c_{0} T_{e}}{2}$, the constant phase term $\frac{k_{r} x_{n} T_{e}^{2}}{2}$ can be ignored. As $x_{n} T_{e}$ is a constant depending on the physical parameters of the array, ignoring $\frac{k_{r} x_{n} d \sin (\theta) T_{e}}{c_{0}}$ will cause deformation in the pulse shapes for larger observation point angles and a shift in the timings. But the deformation can be ignored in analytical derivations and thus it is ignored in the derivations. In this case, after matched filtering, the received array data can be expressed as

$$
\mathbf{y}(t)=\frac{\sigma_{s}^{2}}{r} \mathbf{b}(r, \theta, t)+v(t)
$$

where $v(t)$ is the noise vector and $\mathbf{b}(r, \theta, t)$ is the receive steering vector:

$$
\mathbf{b}(r, \theta, t)=\left[\begin{array}{llll}
1 & e^{j \varphi_{0}(r, \theta, t)} & \ldots & e^{j(N-1) \varphi_{0}(r, \theta, t)}
\end{array}\right]^{T} .
$$

where

$$
\varphi_{0}(r, \theta, t)=-2 \pi f_{0} T_{e}-k_{r} t T_{e}+\frac{2 \pi f_{0} d \sin \theta}{c_{0}}+\frac{k_{r} r_{0} T_{e}}{c_{0}}+\frac{k_{r} d \sin \theta t}{c_{0}}
$$

Suppose there are $D$ sources, the covariance matrix is

$$
\begin{aligned}
\mathbf{R}_{y y} & =E\left\{\mathbf{y}(t) \mathbf{y}^{H}(t)\right\} \\
& =\mathbf{B R}_{s s} \mathbf{B}^{H}+\sigma_{n}^{2} \mathbf{I}_{N}
\end{aligned}
$$

where $E$ represents the expectation operator, ${ }^{H}$ is the conjugate transpose, $\mathbf{B}=\left[\begin{array}{llll}\mathbf{b}\left(r_{1}, \theta_{1}, t\right) & \mathbf{b}\left(r_{2}, \theta_{2}, t\right) & \ldots & \mathbf{b}\left(r_{D}, \theta_{D}, t\right)\end{array}\right]$ is the array manifold matrix, $\mathbf{R}_{s s}$ is the source autocorrelation matrix, $\sigma_{n}^{2}$ is the noise variance and $\mathbf{I}_{N}$ is the $N \times N$ identity matrix. When the sources are temporally uncorrelated, $\mathbf{R}_{y y}$ can be vectorized as [7]

$$
\begin{aligned}
\mathbf{z} & =\operatorname{vec}\left(\mathbf{R}_{y y}\right) \\
& =\sum_{i=1}^{D}\left[\mathbf{b}^{*}\left(r_{i}, \theta_{i}, t\right) \otimes \mathbf{b}\left(r_{i}, \theta_{i}, t\right)\right] \sigma_{i}^{2}+\sigma_{n}^{2} \operatorname{vec}\left(\mathbf{I}_{N}\right) .
\end{aligned}
$$

The $N^{2} \times 1$ vector $\mathbf{z}$ can be seen as the signal received at the difference co-array with the amplitudes of the source signal vector replaced by their corresponding powers. Correspondingly, the equivalent difference co-array steering vector is $\mathbf{u}(r, \theta, t)=\mathbf{b}^{*}(r, \theta, t) \otimes \mathbf{b}(r, \theta, t)$. The distinct elements of $\mathbf{u}(r, \theta, t)$ behave like the manifold of a (longer) array whose element positions are given by the distinct values in the array position set. Therefore, the maximum DOFs for a $N$-element nested array is $N(N-1)+1$.

\section{Beamforming-Based Target Range and Angle Estimation}

Since the virtual data $\mathbf{z}$ of (8) can be seen as the signal received at the difference co-array of nested array receiver with the amplitudes of the source signal vector replaced by their corresponding powers, beamforming can be further applied in the receiver.

Though the incident jammers are originally assumed uncorrelated, after the difference co-array processing, they are represented by their powers and consequently the resulting covariance matrix will be of rank 1. In [5], this problem is tackled with the use of the forward spatial smoothing method at an expense of aperture length loss because it essentially halves the total DOFs offered by the difference co-array, meaning that less sources can be identified. To reduce the aperture length loss, we use a two-way smoothing method based on the forward-backward spatial smoothing technique [8].

We divide the virtual $L$-element co-array into $K$ overlapping subarrays, each with $P$ elements, namely, $L=P+K-1$. That is, the number of smoothing times is $K$. Define two $P \times L$ matrices:

$$
\begin{aligned}
\mathbf{F}_{k} & =\left[\begin{array}{llll}
\mathbf{0}_{P \times(k-1)} & \mathbf{I}_{P} & \mathbf{0}_{P \times(K-k)}
\end{array}\right] \\
\mathbf{G}_{k} & =\left[\begin{array}{llll}
\mathbf{0}_{P \times(k-1)} & \mathbf{J}_{P} & \mathbf{0}_{P \times(K-k)}
\end{array}\right]
\end{aligned}
$$

where $k$ represents the $k$ th smoothing subarray, $\mathbf{0}_{P \times(k-1)} \in$ $\mathbb{R}^{P \times(k-1)}$ and $\mathbf{O}_{P \times(K-k)} \in \mathbb{R}^{P \times(K-k)}$ are zero matrices, and $\mathbf{J}_{P}$ is the $P \times P$ exchange matrix whose anti-diagonal elements are 1 and zero otherwise. Multiplying $\mathbf{F}_{k}$ and $\mathbf{G}_{k}$ by (8) and its conjugate, respectively, yields

$$
\begin{array}{r}
\mathbf{z}_{k}^{f}=\mathbf{F}_{k} \mathbf{z} \\
\mathbf{z}_{k}^{b}=\mathbf{G}_{k} \mathbf{z}^{*}
\end{array}
$$

where $^{*}$ is the conjugate operator. Their covariance matrices are

$$
\begin{array}{r}
\mathbf{R}_{k}^{f}=E\left\{\mathbf{z}_{k}^{f}\left(\mathbf{z}_{k}^{f}\right)^{H}\right\}=\mathbf{F}_{k} E\left\{\mathbf{z z}^{H}\right\} \mathbf{F}_{k}^{H}=\mathbf{F}_{k} \mathbf{R}_{z z} \mathbf{F}_{k}^{H} \\
\mathbf{R}_{k}^{b}=E\left\{\mathbf{z}_{k}^{b}\left(\mathbf{z}_{k}^{b}\right)^{H}\right\}=\mathbf{G}_{k} E\left\{\mathbf{z}^{*} \mathbf{z}^{* H}\right\} \mathbf{G}_{k}^{H}=\mathbf{G}_{k} \mathbf{R}_{z z}^{*} \mathbf{G}_{k}^{H}
\end{array}
$$


The forward and backward spatial smoothing data covariance matrices are expressed, respectively, as

$$
\begin{aligned}
& \mathbf{R}_{z z}^{f}=\frac{1}{K} \sum_{k=1}^{K} \mathbf{R}_{k}^{f} \\
& \mathbf{R}_{z z}^{b}=\frac{1}{K} \sum_{k=1}^{K} \mathbf{R}_{k}^{b}
\end{aligned}
$$

Correspondingly, the forward-backward spatial smoothing data covariance is

$$
\begin{aligned}
\mathbf{R}_{z z}^{f b} & =\frac{1}{2}\left(\mathbf{R}_{z z}^{f}+\mathbf{R}_{z z}^{b}\right) \\
& =\frac{1}{2 K}\left(\sum_{k=1}^{K} \mathbf{F}_{k} \mathbf{R}_{z z} \mathbf{F}_{k}^{H}+\sum_{k=1}^{K} \mathbf{G}_{k} \mathbf{R}_{z z}^{*} \mathbf{G}_{k}^{H}\right) .
\end{aligned}
$$

It has already been proved that when $P \geq D$ and $2 K \geq D$, the forward-backward smoothing data covariance $\mathbf{R}_{z z}^{f b}$ will be of full-rank [8]. We then have

$$
L=P+K-1 \geq D+\frac{D}{2}-1 \Rightarrow D \leq \frac{2(L+1)}{3} .
$$

Therefore, after forward-backward spatial smoothing [9], [10], we can use the full-rank covariance matrix $\mathbf{R}_{z z}^{f b}$ for minimum variance distortionless response (MVDR) beamforming. By eigendecomposing $\mathbf{R}_{z z}^{f b}$ as $\mathbf{R}_{z z}^{f b} \triangleq \mathbf{E}_{s} \boldsymbol{\Lambda}_{s} \mathbf{E}_{s}^{H}+\mathbf{E}_{n} \boldsymbol{\Lambda}_{n} \mathbf{E}_{n}^{H}$, where the diagonal matrix $\boldsymbol{\Lambda}_{s}$ contains the $P$ largest eigenvalues and the columns of $\mathbf{E}_{s}$ are the corresponding eigenvectors, and the diagonal matrix $\boldsymbol{\Lambda}_{n}$ contains the remaining $(L-P)$ eigenvalues and the columns of $\mathbf{E}_{n}$ are the corresponding eigenvectors, the range and angle of targets can be estimated from the $L$ magnitude peaks of the multiple signal classification (MUSIC) estimator [11]:

$$
\begin{aligned}
\{\hat{r}, \hat{\theta}\} & =\arg \max _{r, \theta}|f(r, \theta)|^{2} \\
& =\arg \max _{r, \theta}\left|\frac{\mathbf{b}_{s}^{H}(r, \theta, t) \mathbf{b}_{s}(r, \theta, t)}{\mathbf{b}_{s}^{H}(r, \theta, t) \mathbf{E}_{n} \mathbf{E}_{n}^{H} \mathbf{b}_{s}(r, \theta, t)}\right|^{2} .
\end{aligned}
$$

where $\mathbf{b}_{s}(r, \theta, t)$ is the sub-array steering vector of the form:

$$
\mathbf{b}_{s}(r, \theta, t)=\left[\begin{array}{llll}
1 & e^{j \varphi_{0}(r, \theta, t)} & \ldots & e^{j(P-1) \varphi_{0}(r, \theta, t)}
\end{array}\right]^{T} .
$$

The Cramér-Rao lower bound (CRLB) of target range estimation and angle estimation can be derived with the following data model [5]

$$
\tilde{\mathbf{z}}_{1 i}=\mathbf{B}_{1 i}(r, \theta, t) \sigma_{s}^{2}+\sigma_{n}^{2} \mathbf{e}_{i}^{\prime}
$$

where $\mathbf{B}_{1 i}$ is a $\left(\frac{N^{2}}{4}+\frac{N}{2}\right) \times D$ matrix consists of the $\left(\frac{N^{2}}{4}+\frac{N}{2}+1-i\right)$ th to $\left(\frac{N^{2}-2}{2}+N+1-i\right)$ th rows of $\mathbf{B}_{1}$ which is obtained from $\mathbf{B}^{*} * \mathbf{B}$ by removing the repeated rows (after their first occurrence), and $\mathbf{e}_{i}^{\prime}$ is a vector of all zeros expect a 1 at the $i$ th position. The CRLBs can be obtained as the inverse of the Fisher information matrix (FIM). Specifically, when the angle is known and range is unknown, the range FIM can be derived as

$$
\begin{aligned}
\mathbf{I}_{r r} & =2 \operatorname{Re}\left\{\frac{\partial \mathbf{B}_{1 i}^{H}(r, \theta, t)}{\partial r}\left(\mathbf{C}_{n}^{-1}\right) \frac{\partial \mathbf{B}_{1 i}(r, \theta, t)}{\partial r}\right\} \\
& =4 \mathrm{SNR} \sum_{p=0}^{P-1} \frac{k_{r}^{2} T_{d}^{2} p^{2}}{c_{0}^{2}}
\end{aligned}
$$

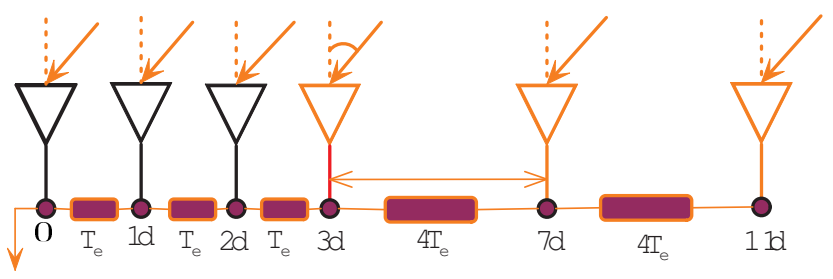

Fig. 2: Illustration of nested array positions.

where SNR denote the signal-to-noise ratio (SNR), namely, $\mathrm{SNR}=\sigma_{s}^{2} / \sigma_{n}^{2}$. Similarly, when the range is known, the angle FIM can be derived as

$$
\begin{aligned}
\mathbf{I}_{\theta \theta} & =2 \operatorname{Re}\left\{\frac{\partial \mathbf{B}_{1 i}^{H}(r, \theta, t)}{\partial \theta}\left(\mathbf{C}_{n}^{-1}\right) \frac{\partial \mathbf{B}_{1 i}(\theta)}{\partial \theta}\right\} \\
& =4 d^{2} \cos ^{2} \theta \cdot \operatorname{SNR}\left(\frac{4 \pi^{2}}{\lambda^{2}}+\frac{k_{r} 4 \pi t}{\lambda c_{0}}+\frac{k_{r}^{2} t^{2}}{c_{0}^{2}}\right) \sum_{p=0}^{P-1} p^{2}
\end{aligned}
$$

\section{Simulation Results}

In the following simulations, we assume that the array with $N_{1}=3$ and $N_{2}=3$ operates at a carrier frequency $f_{0}=10 \mathrm{GHz}, d=\lambda_{0} / 2$ and $T_{e}=5 \mathrm{~ns}$. The array elements are arranged as Figure 2. The additive noise is modeled as a complex Gaussian zero mean spatially and temporally distributed sequence. We compare the proposed nested array with the same array structure FDA denoted as SAS-FDA and the equal aperture length FDA denoted as EAL-FDA.

Suppose there are 5 sources located as the directions $-50^{\circ}$, $-20^{\circ},-5^{\circ}, 10^{\circ}$ and $45^{\circ}$, respectively. Figure 3(a) shows the MUSIC spectra for the three tested arrays. We can see that they all perform well in resolving 5 sources because 6 physical array sensors provide 5 DOFs and thus 5 sources can be well resolved. However, when there are 11 sources, Figure 3(b) shows that the nested array outperforms the EAL-FDA. The latter misses the sources located at $-40^{\circ}$ and $45^{\circ}$, and also has an obvious deviation at $50^{\circ}$. This superior performance is expected since the former enjoys the advantages of nested array in increasing DOFs. Note that, due to its limited DOFs, when there are 11 sources, the MUSIC-based algorithm is not suitable for the SAS-FDA any more and thus no corresponding comparisons are given in Figure 2(b).

Figure 4 compares the range and angle estimation CRLBs versus SNR. It can be seen that the proposed nested array exhibits better CRLBs in both range estimation and angle estimation than the SAS-FDA and EAL-FDA schemes. This is attributed to the reason that the proposed nested array exploits the second-order statistics of the received data with the difference co-array processing algorithm.

\section{Conclusion}

This paper proposed a new receiving array design scheme by nesting two ULAs with diverse time-delayers. It jointly exploits the advantages of nested array in increasing DOFs to resolve more sources and FDA in range-dependent array beampattern in detect range-dependent sources. The diverse time-delayers are used to yield range-dependent receiving array 


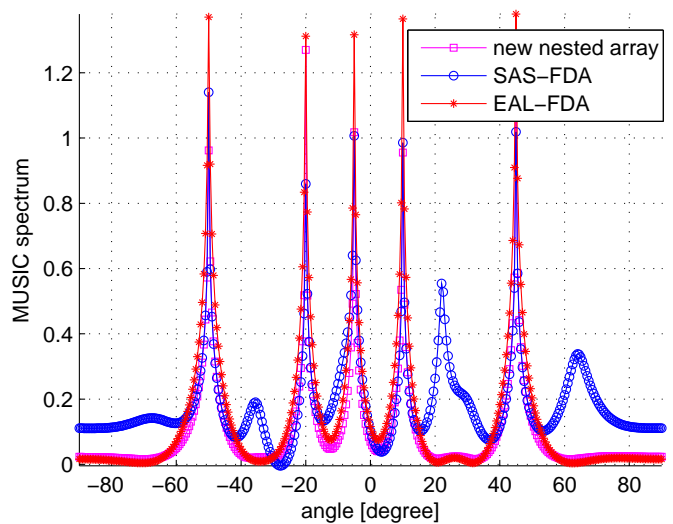

(a) 5 sources.

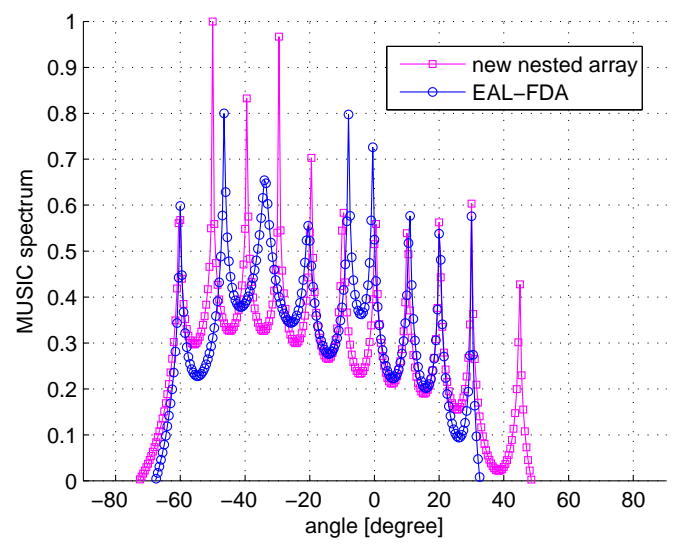

(b) 11 sources.

Fig. 3: Comparisons of MUSIC spectra.

beampattern, which performs similar function as the frequency increments in active transmitting FDA. When the difference co-array processing algorithm is applied in the receiver, much more sources can be resolved in the MUSIC-based range and angle estimator and better (lower) CRLBs can be achieved. Since the proposed array falls into the area of FDA and conventional FDA can only be used in transmitter, this work opens a new avenue to develop new FDA techniques.

\section{ACKNOWLEDGMENT}

The work described in this paper was supported by a Marie Curie Fellowship (FP7/2007-2013, grant agreement PIIF-GA2012-326672).

\section{REFERENCES}

[1] Y. I. Abramovich, N. K. Spencer, and A. Y. Gorokhov, "Positive-definite Toeplitz completion in DOA estimation for nonuniform linear antenna arrays," IEEE Transactions on Signal Processing, vol. 47, no. 6, pp. 1502-1521, June 1999.

[2] M. C. Dogan and J. M. Mendel, "Applications of cumulants to array processing: I. Aperture extension and array calibration," IEEE Transactions on Signal Processing, vol. 43, no. 5, pp. 1200-1216, May 1995.

[3] P. Chevalier and A. Ferreol, "On the virtual array concept for the fourth-oder direction finding problem," IEEE Transactions on Signal Processing, vol. 47, no. 9, pp. 2592-2595, September 1999.

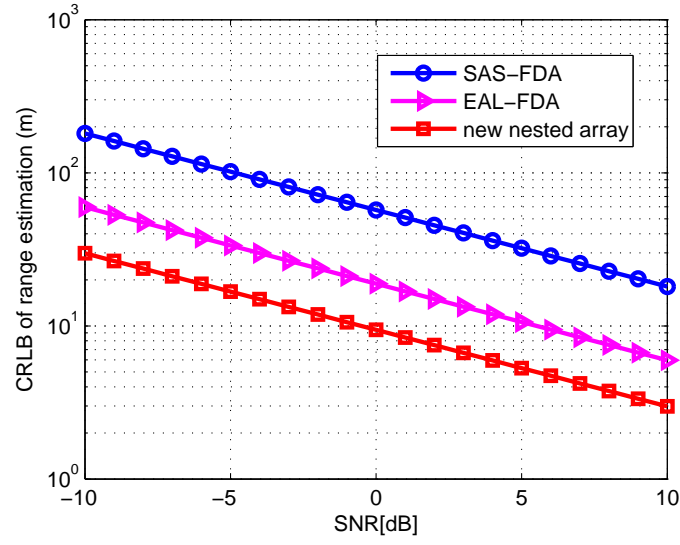

(a) Range CRLB when the angle is known.

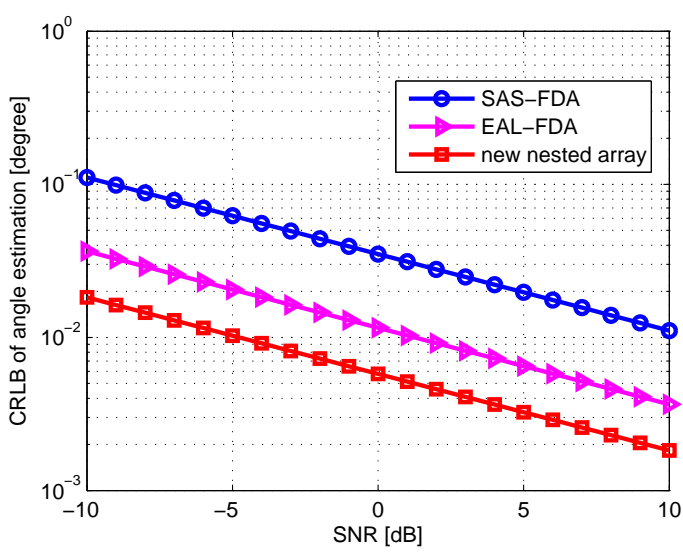

(b) Angle CRLB when the range is known.

Fig. 4: Comparisons of CRLBs versus SNR.

[4] R. T. Hoctor and S. A. Kassam, "The unifying role of the coarray in aperture synthesis for coherent and incoherent imaging," Proceedings of the IEEE, vol. 78, no. 4, pp. 735-752, April 1990.

[5] P. Pal and P. P. Vaidyanathan, "Nested arrays: a novel approach to array processing with enhanced degrees of freedom," IEEE Transactions on Signal Processing, vol. 58, no. 8, pp. 4167-4181, August 2010.

[6] W.-Q. Wang and H. C. So, "Transmit subaperturing for range and angle estimation in frequency diverse array radar," IEEE Transactions on Signal Processing, vol. 62, no. 8, pp. 2000-2011, April 2014.

[7] W. K. Ma, T. H. Hsieh, and C. Y. Chi, "DOA estimation of quasistationary signals with less sensors than sources and unknown spatial noise covariance: A Khatri-Rao subspace approach," IEEE Transactions on Signal Processing, vol. 58, no. 4, pp. 2168-2179, April 2010.

[8] S. U. Pillai and B. H. Kwon, "Forward/backward spatial smoothing techniques for coherent signal identification," IEEE Transactions on Acoustic, Speech, and Signal Processing, vol. 37, no. 1, pp. 8-15, January 1989.

[9] W. Li, Y. Li, and W. Yu, "On adaptive beamforming for coherent interference suppression via virtual antenna array," Progress In Electromagnetics Research, vol. 125, no. 1, pp. 165-184, Februrary 2012.

[10] — - "An effective technique for enhancing anti-interference performance of adaptive virtual antenna array," Applied Computational Electromagnetics Society Journal, vol. 26, no. 3, pp. 234-240, March 2011.

[11] R. O. Schmidt, "Multiple emitter location and signal parameter estimation," in Proceedings of the RADC Spectral Estimation Workshop, Rome, NY, 1979, pp. 234-258. 